\title{
Bioengineering of Stem Cell Microenvironments Using High-Throughput Technologies
}

David A. Brafman*

Cellular and Molecular Medicine, Stem Cell Program, 9500 Gilman Drive, University of California, San Diego, La Jolla, CA 92093, USA

\begin{abstract}
Stem cells represent a ready supply of cellular "raw material" that can be used to study development and disease progression, to perform drug screens, and to treat a variety of degenerative disorders such as Parkinson's, diabetes, and heart disease. Experimental manipulation of these cells to affect self-renewal, proliferation, and differentiation is central to moving these therapies from bench-to-bedside. In vivo, stem cells reside in complex microenvironments where their fate is tightly controlled by a variety of biochemical and physical signals. However, conventional in vitro cell culture methods poorly mimic the in vivo cell microenvironment and are limited in their ability to screen the myriad of factors that can influence stem cell fate. To that end, the development of high-throughput screening (HTS) technologies has allowed for an increased understanding of the microenvironmental factors that govern stem cell fate. In this review, the application of these emerging HTS platforms to understanding the complexities of stem cell biology will be discussed. Furthermore, the advantages, limitations, and potential applications of these technologies will be explored.
\end{abstract}

Keywords: Human embryonic stem cells; Human induced pluripotent stem cells; Stem cell microenvironments; High throughput screening

\section{Introduction}

In contrast to the fully mature cells of the adult body, stem cells are unspecialized cells that possess two unique characteristics-the ability to proliferate indefinitely and to adopt new cellular fates. Therefore, stem cells and their derivatives represent a novel system to study basic biology and human development, screen the safety and efficacy of drugs, and provide unlimited sources of raw material for regenerative medicine therapies to treat human disease and injury. Moreover, human pluripotent stem cells (hPSCs), which can give rise to the hundreds of different cell types that comprise the adult human body, have the potential to revolutionize medicine by creating therapies for many diseases where no effective cures currently exist such as Alzheimer's and heart disease. However, the basic research, pharmaceutical, and therapeutic applications of stem cells remains limited by lack of in vitro methods to reproducibly influence stem cell self-renewal and differentiation. For example, many adult stem cells cannot be easily propagated long-term in vitro and the directed differentiation of hPSCs into mature cell types and tissues remains difficult. As such, much research has been devoted to understanding stem cell behavior and manipulating their differentiation potential as means to developing strategies for the production of defined and functional populations.

In vivo, stems cells reside in a complex microenvironment where their self-renewal and differentiation are tightly controlled (Figure 1). Classical reductionist methods are limited in their ability to study the large number of the chemical, mechanical and physical factors that can influence stem cell fate. Typically, these methods only investigate one factor at a time, thereby ignoring the complex signaling interactions that occur between different components of the microenvironment. As a result, these conventional in vitro techniques poorly resemble the physical, chemical, and biological aspects of the in vivo microenvironment. These limitations have led to advances in utilizing high-throughput screening (HTS) platforms with stem cells as means of better constructing and studying complex in vitro microenvironments. Moreover, these platforms have allowed for the high-throughput study of combinations of various microenvironmental components, including immobilized protein factors (e.g. extracellular matrix proteins, proteoglycans), soluble protein factors (e.g. growth factors, small molecules, hormones), mechanical forces (e.g. stretch, substrate rigidity, shear), and cell-cell interactions (e.g. cadherins, Notch ligands). Research conducted with these technologies has not only led to a clearer understanding of the role of several signaling pathways in controlling stem cell fate but also the development of novel stem cell approaches for regenerative medicine applications.

Here, the various HTS screening platforms that have been applied to studying stem cell biology will be discussed (Figure 2 and Table 1). Although many of these platforms have been applied to stem cells of non-human origin, this review will focus on the applications with human stem populations. Specifically, this review will focus on the following HTS technologies: (1) microtiter platforms, (2) combinatorial protein arrays, (3) biomaterial arrays, (4) micropatterned microwell arrays, and (5) microfluidic platforms. In addition, the advantages and limitations of each of these platforms will be outlined. Finally, future application of these and other HTS technologies to stem cell biology will be discussed.

\section{Components of stem cell microenvironments}

Stem cells have been identified in both developing embryonic and fully mature adult organisms. Generally speaking, stem cells can be categorized into two different populations, which have different properties: (1) Multi- or uni-potent adult stem cells which only give rise to a specialized subset of cell types and (2) Pluripotent stem cells which proliferate indefinitely in an undifferentiated state while retaining their ability to differentiate into all mature cell types. Because

*Corresponding author: David Brafman, Department of Cellular and Molecula Medicine, Stem Cell Program, UCSD, 9500 Gilman Drive, La Jolla CA 92093-0395, USA, E-mail: dbrafman@ucsd.edu

Received November 30, 2011; Accepted February 15, 2012; Published February 18,2012

Citation: Brafman DA (2012) Bioengineering of Stem Cell Microenvironments Using High-Throughput Technologies. J Bioengineer \& Biomedical Sci S5:004. doi:10.4172/2155-9538.S5-004

Copyright: (C) 2012 Brafman DA. This is an open-access article distributed under the terms of the Creative Commons Attribution License, which permits unrestricted use, distribution, and reproduction in any medium, provided the original author and source are credited. 
Citation: Brafman DA (2012) Bioengineering of Stem Cell Microenvironments Using High-Throughput Technologies. J Bioengineer \& Biomedical Sci S5:004. doi:10.4172/2155-9538.S5-004

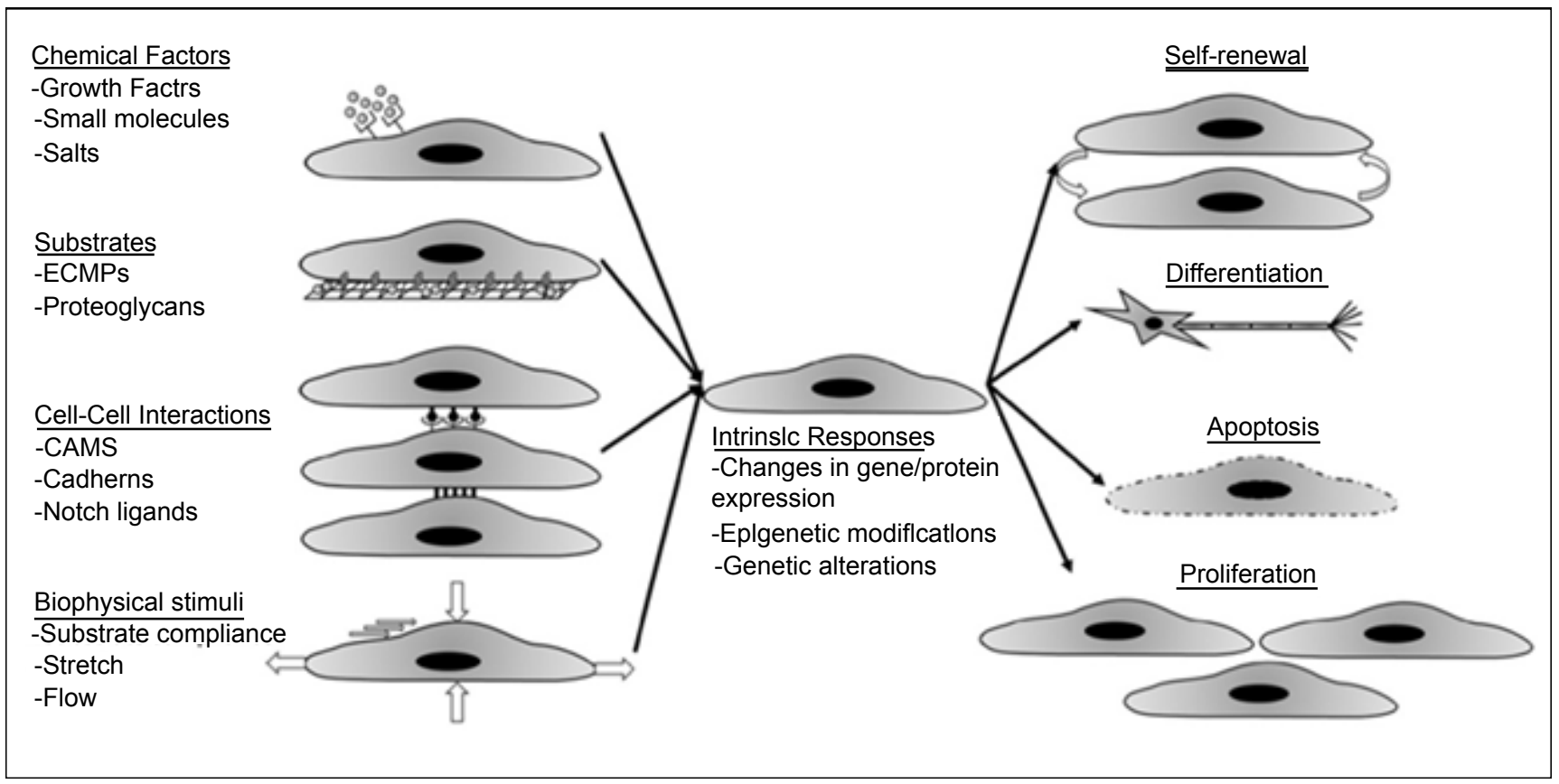

Figure 1: Components of the stem cell microenvironment. Microenvironmental cues such as soluble signaling protein factors (e.g. growth factors, small molecules), immobilized substrates (e.g. extracellular matrix proteins, proteoglycans), cell-cell interactions (e.g. cell adhesion molecules, cadherins, Notch ligands), and mechanical forces (e.g. substrate rigidity, flow, stretch) communicate in a complex manner to influence various signaling pathways, and ultimately affect cell fate and function.

\begin{tabular}{|c|c|c|c|c|c|}
\hline Platform & Microtiter Platforms & $\frac{\frac{\text { Combinatorial }}{\text { Protien Arrays }}}{- \text { ECMPs, GAGs }}$ & Biomaterial Arrays & $\begin{array}{l}\text { Micropatterned } \\
\text { Microwell Arrays }\end{array}$ & 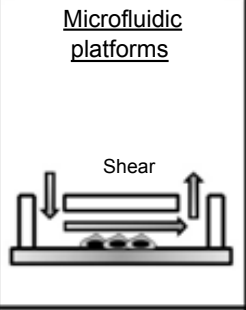 \\
\hline Advantages & $\begin{array}{l}\text {-Unbiased } \\
\text {-Ability to screen } \\
\text { large, diverse } \\
\text { libraries } \\
\text { •High-throughput }\end{array}$ & $\begin{array}{l}\text {-Ability to screen } \\
\text { multiple } \\
\text { components } \\
\text { simultaneously } \\
\text { - Miniaturized assays }\end{array}$ & $\begin{array}{l}\text { - Ability to screen } \\
\text { defined, synthetic } \\
\text { substrates } \\
\text { - Miniaturized assays }\end{array}$ & $\begin{array}{l}\text { - Ability to interrogate } \\
\text { biophysical properties } \\
\text { - Precise control over } \\
\text { seeding density }\end{array}$ & $\begin{array}{l}\text { - Ability to apply shear } \\
\text { forces } \\
\text {-Simultaneous control } \\
\text { over soluble and } \\
\text { mechanical properties }\end{array}$ \\
\hline Limitations & $\begin{array}{l}\text { Large number of } \\
\text { cells required } \\
\text { - Off-target effects } \\
\text { - Interrogate one } \\
\text { factor at a time }\end{array}$ & $\begin{array}{l}\text {-Rely on purified or } \\
\text { recombinant } \\
\text { proteins } \\
\text {-Ignore biophysical } \\
\text { properties }\end{array}$ & $\begin{array}{l}\text {-Non-physiological } \\
\text { substrate } \\
\text {-Not combinatorial }\end{array}$ & \begin{tabular}{|l|} 
- Low throughput \\
- Difficult to vary other \\
microenvironmental \\
variables
\end{tabular} & $\begin{array}{l}\text { - Low throughput } \\
\text {-Inability to perform } \\
\text { on-chip } \\
\text { immunocytochemistry } \\
\text { - Not compatible w/ } \\
\text { conventional } \\
\text { microscopy }\end{array}$ \\
\hline $\begin{array}{c}\text { Key } \\
\text { Examples } \\
\end{array}$ & $\begin{array}{l}\text {-Small molecules } \\
\text { that promote hPSC } \\
\text { self-renewal }(33,44) \\
\text { Compounds that } \\
\text { direct hPSC } \\
\text { differentiation (36- } \\
\text { 39) }\end{array}$ & $\begin{array}{l}\text { - Proliferation and } \\
\text { differentiation of } \\
\text { NPCs (43) } \\
\text { - Expansion of } \\
\text { mammary } \\
\text { progenitors (44) } \\
\text {-Self-renewal and } \\
\text { differentiation of } \\
\text { hPSCs (45) }\end{array}$ & $\begin{array}{l}\text { •Polymers that } \\
\text { influence hMSC fate } \\
(51,52) \\
\text {-Synthetic substrate } \\
\text { for expansion of } \\
\text { hPSCs }(53,54) \\
\text { - Differentiation of } \\
\text { hPSCs }(50,55)\end{array}$ & $\begin{array}{l}\text { HSC expansion (59) } \\
\cdot \text { Neurosphere } \\
\text { expansion (60) } \\
\cdot \text { Create hPSC EBs of } \\
\text { homogenous size } \\
\text { and shape }(61-64)\end{array}$ & $\begin{array}{l}\text {-Effect of shear and } \\
\text { soluble factors on } \\
\text { stem cell fate }(66,67) \\
\text { - Influence of } \\
\text { morphogen gradient } \\
\text { on NPC fate }(68)\end{array}$ \\
\hline
\end{tabular}

Figure 2: High-throughput platforms for investigating stem cell fate. Each platform has several advantages as well as limitations. Additionally, each platform has widely been used to study stem cell biology.

Abbreviations: ECMPs: Extracellular Matrix Proteins; GAGs: Glycoaminoglycans; GFs: Growth Factors; SMs: Small Molecules; hPSCs: Human Pluripotent Stem Cells; NPCs: Neural Progenitor Cells; HSC: Hematopoietic Stem Cell. 


\begin{tabular}{|c|c|c|c|}
\hline Platform Used & Stem Cell Studied & Key Findings & Reference \\
\hline \multirow[t]{5}{*}{ Microtiter } & hPSC & Small molecule (Y-27632) promotes hPSC single cell survival & [31] \\
\hline & DE & Developed 5 compound cocktail that promotes hPSC survival & [32] \\
\hline & PP & Identified four compounds to promote hPSC self-renewal & [33] \\
\hline & $\mathrm{CM}$ & EHNA maintains long-term hPSC pluripotency & [34] \\
\hline & NESCS & PEDF, signaling through the Erk1/2 signaling pathway, sustains long-term maintenance of NESCs & [35] \\
\hline \multirow[t]{3}{*}{ Combinatorial Protein Array } & hPSCs & Defined ECMP for growth of hPSCs & [45] \\
\hline & MPCs & $\begin{array}{l}\text { Laminin-1 maintained MPCs in a quiescent state while P-cadherin directed differentiation into a } \\
\text { myoepithelial cell type }\end{array}$ & [44] \\
\hline & NPCs & $\begin{array}{l}\text { Wnt and Notch co-stimulation maintain NPCs in a multipotent state whereas BMP-4 induces differen- } \\
\text { tiation into neuronal and glial fates }\end{array}$ & [43] \\
\hline \multicolumn{4}{|l|}{ Biomaterial Array } \\
\hline & hPSCs & $\begin{array}{l}\text { Substrates supported optimal hPSC growth have moderate wettability and relied on integrin aVB3 and } \\
\text { avB5 engagement with adsorbed Vn to promote hPSC expansion }\end{array}$ & [53] \\
\hline & hPSCs & Heparin mimicking polymer supports long-term hPSC growth of multiple hPSC lines & [54] \\
\hline & ECs & Differentiation of hPSCs into ECs & {$[50]$} \\
\hline & HE & Novel substrates for the generation of $\mathrm{HE}$ from hPSCs & [55] \\
\hline & MSCs & $\begin{array}{l}\text { Phosphate surfaces promote osteoblast formation while t-butyl-modified surfaces direct adipocyte } \\
\text { formation }\end{array}$ & [51] \\
\hline \multirow[t]{4}{*}{ Microwell Array } & hPSCs & Small EBs form neuroectoderm whereas large EBs tend towards a mesoendoderm fate & [63] \\
\hline & & $\begin{array}{l}\text { EB size regulates the amount of endothelial and cardiac differentiation in hPSCs through differential } \\
\text { Wnt signaling }\end{array}$ & [64] \\
\hline & HSCs & Proliferation and differentiation of HSCs decreases in microwells of smaller size & {$[58]$} \\
\hline & NPCs & Viability of neurospheres enhanced by controlling their size & [60] \\
\hline \multicolumn{4}{|l|}{ Microfluidic } \\
\hline & hPMPCs & Combination of shear stress and VEGF increases endothelial differentiation of hPMPCs & [66] \\
\hline & NPCs & Differentiation of NPCs directly proportion to Shh concentrations in the gradient & [68] \\
\hline
\end{tabular}

Table 1: Examples of uses of high-throughput platforms for investigating stem cell microenvironments.

Abbreviations: hPSCs: Human Pluripotent Stem Cells; EHNA: [erythro-9-(2-hydroxy-3-nonyl)adenine]; PEDF: Pigment Epithelium-Derived Factor; DE: Definitive Endoderm; ILV: (-)-indolactam-V; PP: Pancreatic Progenitors; CMw: Cardiomyocytes; NESCs: Neuralepithelial Stem Cells; MPCs: Mammary Progenitor Cells; NPCs: Neural Progenitor Cells; Vn: Vitronectin; ECs: epithelial cells; HE: Hepatic Endoderm; MSCs: Mesenchymal Stem Cells; EB: Embryoid Body; HSCs: Hematopoietic Stem Cells; hPMPCs: Human Placenta-Derived Multipotent Cells.

most adult stem cell types are extremely rare and difficult to isolate and maintain, most HTS technologies have made use of pluripotent stem cell alternatives.

In vivo, adult stem cells reside in local tissue microenvironments called niches that regulate the balance between self-renewal and differentiation $[1,2]$. In all cases, the niche components maintain a delicate homeostatic balance to avoid excessive self-renewal, which could lead to cancer, and differentiation, which could lead to depletion of a tissue's regenerative potential [3]. The first stem cell niche identified was the hematopoietic stem cell (HSC) niche [4]. More recently, additional stem cell niches have been located under the basal lamina on myofibers (muscle satellite cells), subventricular zone of the lateral ventricle of the central nervous system (neural stem cells; NSCs), bulge region of the hair follicle (epithelial stem cells), basal layer of the epidermis (epidermal stem cells), basal layer of the seminiferous tubules (germline stem cells) and the base of the crypt of the intestinal epithelium [5-10]. HSCs are multipotent and highly self-renewing in vivo but difficult to maintain and expand in vitro [11]. On the other hand, several other stem populations have been isolated and propagated successfully in vitro. For example, long-term culture of adult human neural progenitor cells has been successful with exogenous factors such as basic fibroblast growth factor (bFGF) and epidermal growth factor (EGF) [12].

The niches of the various tissues of the adult body share certain physical and organizational components and properties that regulate the regenerative properties of the stem cells that reside within. The physical cell-cell interactions provided by the niche cells regulate the self-renewal of the neighboring stem cells. For example, the mesenchymal myofibroblasts and the epithelial cells of the intestinal niche form a physical crypt structure that regulates stem cell selfrenewal, migration, and differentiation [13]. Similarly, Notch signaling maintains NSC multipotency and inhibits neurogenesis [14]. Growth factors and other signaling molecules also contribute to determining stem cell fate within the niche. In the spermatogonial stem cell niche, the sertoli cells, which are the main component of the niche, produce glial cell line-derived neurotrophic factor (GDNF) that maintains spermatogonial stem cell self-renewal [15]. Furthermore, soluble signals from outside of the niche can also influence stem cell behavior. For instance, stromal-derived factor-1 (SDF-1) secreted by injured cells outside of the niche play a critical role in the homing of hematopoietic stem cells in bone marrow [16]. Cell-extracellular matrix protein (ECMP) interactions also play a critical role in regulating stem cell fate within the niche. The ECMP not only provides a scaffold for stem cell growth but also interacts with soluble factors to regulate signal transduction. As an example, Stier et al. [17] identified a matrix protein, osteopontin (OPN), as a negative regulator of hematopoietic stem cell expansion within the bone marrow niche. Along similar lines, glycoaminoglycans can influence stem cell behavior within the niche by locally concentrating and presenting soluble growth factors. Recently, it was demonstrated that heparan sulfate proteoglycans regulate BMP signaling in the germ line stem cell niche [18,19]. Finally, other physiochemical factors, including oxygen gradients, $\mathrm{pH}$, matrix stiffness, and topography have been shown to regulate stem cell selfrenewal, proliferation, and differentiation within the niche [20]. 
Human pluripotent stem cells (hPSCs) include both human embryonic stem cells (hESCs) and induced pluripotent stem cells (hiPSCs). HESCs are derived from the inner cell mass of a preimplantation blastocyst [21]. On the other hand, hiPSCs are derived from the ectopic expression of four transcription factors KLF4, OCT4, c-MYC, and SOX2 in mature and specialized cell types [22]. Both of these cell types are able to proliferate indefinitely in culture and generate all derivatives of the three primary germ layers-ectoderm, endoderm, and mesoderm. Although hESCs do not have a stable niche in vivo, various in vitro culture conditions for hPSC proliferation and differentiation have been developed. Specifically, it has been demonstrated that hPSC fate can be controlled by a variety of factors including ECMPs, soluble factors, substrate rigidity, oxygen tension, and mechanical forces [23].

\section{Microtiter HTS to identify novel modulators of human stem cell fate}

Typically, traditional HTS uses 96 or 384 well microtiter plates combined with automated high-content imagining to screen diverse libraries consisting of hundreds of thousands of small molecules, in order to identify compounds that perturb a specific set of signaling pathways and, therefore, produce a desired phenotype [24]. Traditional HTS is an iterative process in which initial primary screens are used to identify 'hit' compounds that generate the phenotype of interest, and secondary assays are implemented to confirm the compound activity, elucidate the mechanism of action, and interrogate chemical structurebiological activity relationships $[25,26]$. Traditional HTS has been used by several groups to identify various bioactive compounds and pathways that affect human stem cell fate.

Although many groups have used HTS of chemical libraries to identify small molecules that regulate mouse ESC (mESC) self-renewal [27] or differentiation into multiple lineages [28-30], the development of HTS for hPSCs has been challenging because of difficulties in establishing suitable plating and growth conditions in miniaturized formats. Specifically, hPSCs are propagated as aggregated colonies and suffer poor survival after single cell dissociation, which is required for most HTS. Therefore, one of the earliest HTS with hPSCs was conducted to identify compounds that permit survival of dissociated hPSCs and allow for their clonal expansion [31]. Specifically, Watanabe et al. [31] identified a Rho-associated kinase (ROCK) inhibitor, Y-27632, which significantly decreased single cell-induced apoptosis of hPSCs. Along similar lines, a screen of 1040 compounds identified 5 compounds that promote hPSC survival and revealed that activity of PRK2, ROCK, MNK1, RSK1, and MSK1 kinases regulate the survival of dissociated hESCs [32].

Several studies have implemented traditional HTS of compound libraries to identify molecules that promote hPSC self-renewal. Desbordes et al. [33] screened 2880 biologically active compounds for their effects on hPSC self-renewal as measured by maintenance of expression of OCT4, a marker of pluripotency. Four compounds were able to support short-term hPSC self-renewal in the absence of exogenous mitogenic signaling molecules, such as b-FGF, or mouse embryonic fibroblast conditioned media. Yet, none of the identified compounds were able to support the long-term proliferation of hPSCs. On the other hand, a recent HTS of a small molecule library led to the discover of a compound, EHNA [erythro-9-(2-hydroxy-3-nonyl) adenine], that was able to maintain long-term hPSC pluripotency in the absence of exogenous cytokines [34]. Using a similar screening assay, Gonzalez et al. [35], screened over 800 purified secreted proteins on the maintenance of hPSC pluripotency. From this screen, it was discovered that pigment epithelium-derived factor (PEDF), signaling through the Erk1/2 signaling pathway, could sustain long-term maintenance of hPSCs without bFGF or TGF $\beta /$ Activin/Nodal ligand supplementation.

Traditional HTS has also been employed to identify compounds that direct differentiation of hPSCs. For example, small molecule HTS approaches were used to identify a compound, stauprimide, which down regulates $\mathrm{c}-\mathrm{Myc}$ and thus increases the efficiency of the spontaneous differentiation of hPSCs [36]. A similar approach was used to identify four natural compounds, selegiline, cymarin, sarmentogenin, and retinoic acid, which were able to induce a concentration-dependent decrease in OCT4 expression [33]. More recently, traditional HTS strategies have been implemented to identify compounds that direct differentiation of hPSCs towards specific lineages. For example, Borowiak et al. carried out a high-content image-based screen to identify two compounds, IDE-1 and IDE-2, which induce differentiation of hPSC towards definitive endoderm through activation of Nodal/Smad signaling [37]. Building on this work, Chen et al. [38] used a similar strategy to identify a small molecule, (-)-indolactam-V (ILV), which through activation of protein kinase $\mathrm{C}$ (PKC) signaling, efficiently directed the differentiation of hPSCs into PDX1 expressing pancreatic progenitors. Along similar lines, Willems et al. [39] screened 550 known pathway modulators using a high-content assay in which the promoter of cardiac-specific gene MYH6 drove expression of a red fluorescent protein. From this screen, several small molecule inhibitors of the Wnt pathway were identified to promote robust cardiomyocyte differentiation from hPSC derived mesoderm. Finally, a HTS paradigm was implemented to screen a library of commercially available kinase inhibitors to identify compounds that promote the survival, selfrenewal, and expansion of hPSC neuralepithelial stem cells [40].

As these studies show, HTS of chemical libraries to identify modulators of stem cell fate has advantages over traditional reductionist approaches of screening single factor modulators of known target pathways. Specifically, unbiased HTS allows for the identification of novel genes and pathways that control stem cell fate. Despite these advantages, traditional HTS is not practical for all stem cell related investigations due to the cost and the large number of cells and reagents required. For example, most HTS require approximately $5 \times 10^{5}$ cells per condition screened, which is not feasible for screens involving some rare and difficult to isolate adult stem cells. Furthermore, most small molecules screened using HTS modulate multiple signaling pathways and have many off-target effects which make them difficult to use when consistent control over cell phenotype is required. Finally, most HTS approaches only have the capacity to interrogate one factor at a time, thereby ignoring the complex crosstalk that typically occurs in biological systems between combinations of molecules.

\section{Combinatorial protein microarrays for engineering stem cell microenvironments}

In vivo, stem cells are exposed to a variety of factors such as immobilized ECMPs, soluble signaling molecules, and mechanical forces. Each of these factors interacts in a complex manner to influence each other's signaling ability. Combinatorial protein microarrays have been developed in order to understand the complexity of these interactions. Typical combinatorial microarrays consist of an inert substrate (e.g. poly-dimethylsiloxane [PDMS] or functionalized glass microscope slides) where small volumes of biological signaling molecules have been deposited in defined locations. These arrays can then subsequently be analyzed for their effects of cellular processes such as changes in gene or protein expression levels. Early combinatorial 
arrays were used to interrogate the effect of combinations of ECMPs and soluble growth factors on mESC differentiation towards early hepatic [41] and cardiac fates [42]

More recently, combinatorial arrays have been used to interrogate the effect of microenvironment components of human adult stem cell fate. For example, Soen et al. [43] used robotic spotting techniques to print arrays of laminin along with growth factors and cell adhesion molecules to evaluate their effects on the proliferation and differentiation of human adult neural precursor cells. Their analysis revealed significant effects of specific signaling molecules on the extent and differentiation into neuronal or glial fates. For example, Wnt and Notch co-stimulation maintained the neural precursor cells in a multipotent state whereas BMP-4 induced differentiation into neuronal and glial fates. Along similar lines, LaBarge et al. [44] implemented combinatorial arrays to systematically screen pairs of ECMPS and signaling models for the maintenance and differentiation of human mammary gland progenitor cells. The screen revealed that laminin-1 maintained progenitors in a quiescent state while P-cadherin directed differentiation into a myoepithelial cell type.

Combinatorial protein arrays have also been adapted to study the effects of ECMPs and signaling molecules on hPSC fate. Building upon previous combinatorial array technologies, an integrated array platform was developed in which ECMPs, growth factors, and small molecules were non-covalently arrayed on acrylamide-coated slides, thereby creating comprehensive microenvironments that closely mimic the in vivo microenvironment in which stem cells reside [45]. This technology platform was used to screen thousands of unique microenvironments on hPSC attachment, proliferation, and differentiation. Moreover, through the systematic screening of ECMPs and other signaling molecules, a completely defined substrate composed of purified human collagen I, collagen IV, fibronectin, and laminin that supported the culture of three independent hPSC lines in defined media conditions was developed.

Although the throughput of these systems is significantly lower than conventional HTS systems, combinatorial protein microarrays allow for the in vitro interrogation of stem cell microenvironments that closely resemble their in vivo counterparts. Moreover, these studies demonstrate that the ability to build complex stem cell microenvironments one component at a time will reveal interactions between signaling pathways that would not be identified using traditional, low-throughput multi-well assays. On the other hand, combinatorial microarrays often screen animal-based or recombinant proteins which are expensive, difficult to isolate, and subject to batch-to-batch variations. Therefore, conditions identified from combinatorial array screens may be difficult to scale and unsuitable for cell-based therapies.

\section{Biomaterial arrays for identifying synthetic extracellular matrices that control stem cell fate}

Biomaterials have been used as synthetic substrates for the culture of many human adult stem cell and progenitor populations, including mesenchymal stem cells (MSCs; [46-48]) and neural progenitor cells (NPCs; [49]). Nevertheless, development of biomaterials for the culture and differentiation of human stem cells has been limited due to the iterative nature of biomaterials-related research in which polymers are synthesized, tested, modified, and then retested [50]. Similar to combinatorial protein arrays, biomaterial arrays consist of immobilized polymers spotted on a surface onto which cells are seeded. Attached cells are subsequently analyzed for changes in phenotype.
Arrays of polymers have been successfully used to identify specific biomaterial properties that influence stem cell fate. For example, one study implemented an array-based biomaterial screen to identify certain functional groups that direct human mesenchymal stem cell (hMSC) differentiation [51]. With this method, it was elucidated that phosphate surfaces promoted osteoblast formation while t-butylmodified surfaces directed adipocyte formation. A similar technology has been used to identify biodegradable polymers that support the growth and expansion of hMSCs and neural stem cells [52].

More recently, biomaterial arrays have been used to identify synthetic substrates that support hPSC self-renewal and expansion. Using biomaterial arrays, Anderson et al. [52] screened hundreds of polymers with varying wettability, surface topography, surface chemistry and elastic modulus to identify the chemical and material properties that support the long-term culture of hPSCs [53]. From these experiments, a specific polymer chemistry was found to support undifferentiated hPSC growth while elastic modules and surface roughness had a smaller effect on hPSC proliferation. More specifically, the substrates that supported optimal hPSC growth had moderate wettability and relied on integrin $\alpha \mathrm{V} \beta 3$ and $\alpha v \beta 5$ engagement with adsorbed vitronectin to promote hPSC expansion. Along similar lines, a polymer array technology was used to identify a heparin mimicking, polymer, poly (methyl vinyl ether-alt-maleic anhydride) (PMVE-altMA), that supported the long-term attachment, proliferation and self-renewal of multiple hPSC lines [54]. Furthermore, this study revealed that certain biomaterial properties, such as molecular weight, significantly influence hPSC growth and attachment. Biomaterial arrays have also been used to identify polymers that direct differentiation of hPSCs. For example, an array-based approach was implemented to study the effects of 576 synthetic materials on stem cell differentiation [50]. Several classes of biomaterials promoted differentiation of hPSCs into epithelial cells. A similar technology was used for the unbiased screening of polymer libraries to define novel substrates for the generation of hepatic endoderm from hPSCs [55].

Biomaterial arrays have allowed for the identification of substrates that closely mimic the ECMPs found in many stem cell microenvironments. Polymers discovered from these studies will allow for the development of cost-effective, biomaterial-based matrices that support long-term self-renewal, proliferation, and differentiation of stem cells. Furthermore, these synthetic substrates will not only help to accelerate the translational perspectives of stem cells, but also provide a platform to elucidate the underlying molecular mechanisms that regulate stem cell proliferation and differentiation.

\section{Microwell arrays for investigating biophysical cues that influence stem cell fate}

The previously described platforms do not examine the biophysical properties of the stem cell microenvironment which significantly affect stem cell phenotypes. For example, these platforms utilize substrates such as plastic or glass that have nonphysiological mechanical and topographical properties that are not easily modified [56]. Along similar lines, cell density is another microenvironmental parameter that is not readily studied using these platforms. On the other hand, microwell arrays, which can be fabricated with defined physical properties, can overcome these limitations. Moreover, microwell arrays allow for the interrogation of stem cell populations to microenvironmental cues such as soluble mitogens, cell-cell interactions, and cell-substrate interactions. Microwell arrays, which are typically fabricated by using soft lithography methods, have hundreds to thousands of small wells 
arranged in a regular pattern. The dimension of the microwells can be tightly controlled and range from tens to hundreds of micrometers in height and diameter. Cells are captured in the wells by gravitational sedimentation and, therefore, the number of cells trapped in each well can be controlled by the cell seeding density and microwell diameter [57].

Microwell arrays have been used extensively to investigate the influence of physical and structure parameters on adult stem cell fate. For example, fibronectin coated microwells were used to investigate the influence of spatial and adhesive interactions on hematopoietic stem cell fate decisions [58]. Interestingly, proliferation and differentiation of hematopoietic stem cells decreased in microwells of smaller size. Similarly, Lutolf et al. [59] utilized poly (ethylene glycol) (PEG) microwell arrays for the high-throughput study of the effect of cell density, substrate rigidity, and soluble proteins on hematopoietic stem cell fate. Microwell arrays have also been used to assess NSC fate and neurosphere formation [60]. Using this technology, the authors were able to enhance the viability of neurospheres formed by controlling their size from a single founding cell.

Directed differentiation of hPSCs through formation of embryoid bodies, spheroid aggregates that resemble the early stages of embryonic development, has been enhanced through the use of microwell arrays. Traditional methods of embryoid body formation (e.g. suspension aggregation or hanging drop method) are not scalable, produce embryoid bodies heterogeneous in shape and size, and result in variable differentiation. In order to provide more uniform microenvironments to embryoid bodies and thus more consistently direct embryoid body differentiation, microwell approaches have been implemented. For example, PEG and PDMS microwell arrays have been used to create embryoid bodies of homogenous size and shape [61-63]. Moreover, it has been demonstrated that embryoid body size has a significant effect on hPSC differentiation trajectory. For example, Bauwens et al. [63] found that small embryoid bodies were biased towards a neuroectoderm fate whereas large embryoid bodies tended towards a mesoendoderm fate. Along similar lines, Hwang et al. [64] demonstrated embryoid body size regulated the amount of endothelial and cardiac differentiation in hPSCs through differential Wnt signaling.

\section{Studying stem cell biology with high-throughput microfluidic platforms}

Most high-throughput platforms rely on the static exposure of stem cells to microenvironmental components. In turn, these platforms ignore the effects of both shear fluid forces and concentration gradients of biomolecules that influence stem cell fate in vivo. On the other hand, microfluidic platforms allow for the ability to control the soluble and mechanical properties of the stem cell culture environment [65]. Additional advantages of microfluidic over static platforms include decreased reaction rates and analysis times, reduced consumption of reagent, and the ability to run parallel experiments on a single chip. The majority of these platforms are fabricated by using soft lithography methods in which PDMS is casted over a prefabricated mold.

Microfluidic platforms have allowed for the simultaneous investigation of both shear forces and soluble factors on stem cell phenotypes that would be difficult to achieve with other high throughput platforms. For example, Wu et al. [30] investigated the synergism between biochemical and mechanical stimuli in the differentiation of human placenta-derived multipotent cells [66]. The authors found that the combination of shear stress and VEGF increases endothelial differentiation of these cells. A similar platform was used to identify a relationship between variable levels of hydrodynamic shear and the differentiation of hPSCs into vascular lineages [67]. Microfluidic devices have also been used extensively to generate gradients of morphogens to study stem cell behavior. For example, Park et al. [68] implemented a microfluidic device to study the influence of Shh, FGF8, and BMP4 on hPSC derived NPC self-renewal and differentiation. It was revealed that differentiation of the NPCs was directly proportional to Shh concentrations in the gradient.

While microfluidic platforms allow for the multiplexing of experiments and the interrogation of relationships between microenvironmental factors, they have previously been limited by their relative low throughput. Cosson et al. [69] have addressed this limitation by developing a microfluidic device that allows for the high throughput study of overlapping gradients of multiple proteins. Although this technology has only been applied to primary human fibroblasts, its use can easily be extended to enable the high-throughput interrogation of signaling molecule gradients on stem cell fate.

\section{Future Outlook and Concluding Remarks}

Although the technology platforms presented here have allowed for the high-throughput study of various components of the stem cell microenvironment, additional factors such as matrix stiffness, substrate topography, and dimensionalities, which also play critical roles in the determination of cell fate, have yet to be explored in a high-throughput manner. Substrate compliance has been shown to influence the fate of both adult stem cells and hPSCs. For example, lineage specific differentiation of MSCs is aided by substrate stiffness that matches the respective tissue [70]. Specifically, when MSCs were grown on soft matrices that mimic those of the brain, neurogenic markers were upregulated whereas when MSCs were grown on stiff matrices that mimic those of bone, osteogenic markers were upregulated. A similar study demonstrated that substrate modulus directs NSC behavior with softer substrates favoring neural differentiation and harder substrates promoting glial differentiation [71]. Along similar line, in vivo cells often reside in three dimensional as opposed to two dimensional microenvironments [72]. Moreover, three dimensional culture systems have proved an effective means of differentiating stem cells into functionally mature cells such as cardiomyocytes and hepatocytes $[73,74]$.

Because current HTS platforms are unable to investigate the simultaneous effects of substrate rigidity, dimensionality, and other microenvironmental components on stem cell fate, new technologies have recently been developed to overcome these limitations. To that end, Gobaa et al. [56] merged combinatorial protein array and microwell technologies to create a new platform composed of artificial niche microarrays. This technology consisted of hydrogel microwell arrays of varying stiffness where each microwell was functionalized with combinations of proteins spotted by robotic microcontact printing technology [56]. This platform was utilized to investigate the combinatorial effect of cell density, substrate stiffness, ECMPs, and soluble signaling molecules on adipogenic and osteogenic differentiation of human MSCs. Similarly, Yang et al. [75] developed a combinatorial array of three dimensional extracellular matrix proteins to probe the osteogenic and endothelial differentiation of hPSCs. In the future, these emerging technologies will be critical in developing more in-vivo-like HTS conditions to better understand the intricacies of stem cell microenvironments.

In sum, adult stem cells and hPSCs represent the "building blocks" that can be used to engineer more realistic in vitro disease models 
and develop regenerative medicine therapies. However, the fate of these cells can be influenced in a combinatorial manner by numerous physical, biological, and chemical microenvironmental factors. The multifactorial technology platforms presented in this review provide a means for deconstructing the various components that influence stem cell behavior. Moving forward, these HTS systems will allow for the precise control of stem cell phenotypes that will be necessary for the generation of large amounts of defined and mature cell types required for regenerative medicine applications.

\section{References}

1. Fuchs $E$, Tumbar T, Guasch $G$ (2004) Socializing with the neighbors: stem cells and their niche. Cell 116: 769-778.

2. Morrison SJ, Spradling AC (2008) Stem cells and niches: mechanisms that promote stem cell maintenance throughout life. Cell 132: 598-611.

3. Adams J, Adler C, Aggarwal MM, Ahammed Z, Amonett J, et al. (2004) Multistrange baryon production in Au-Au collisions at sqrt $[s(\mathrm{NN})]=130 \mathrm{GeV}$. Phys Rev Lett 92: 182301.

4. Adams GB, Scadden DT (2006) The hematopoietic stem cell in its place. Nat Immunol 7: 333-337.

5. Collins CA, Partridge TA (2005) Self-renewal of the adult skeletal muscle satellite cell. Cell Cycle 4: 1338-1341.

6. Gage FH (2000) Mammalian neural stem cells. Science 287: 1433-1438.

7. Cotsarelis G, Sun TT, Lavker RM (1990) Label-retaining cells reside in the bulge area of pilosebaceous unit: implications for follicular stem cells, hair cycle, and skin carcinogenesis. Cell 61: 1329-1337.

8. de Rooij DG (2001) Proliferation and differentiation of spermatogonial stem cells. Reproduction 121: 347-354

9. Clayton E, Doupé DP, Klein AM, Winton DJ, Simons BD, et al. (2007) A single type of progenitor cell maintains normal epidermis. Nature 446: 185-189.

10. Barker N, van Es JH, Kuipers J, Kujala P, van den Born M, et al. (2007) Identification of stem cells in small intestine and colon by marker gene Lgr5. Nature 449: 1003-1007

11. Li L, Xie T (2005) Stem cell niche: structure and function. Annu Rev Cell Dev Biol 21: 605-631.

12. Gage FH, Ray J, Fisher LJ (1995) Isolation, characterization, and use of stem cells from the CNS. Annu Rev Neurosci 18: 159-192.

13. Yeung TM, Chia LA, Kosinski CM, Kuo CJ (2011) Regulation of self-renewa and differentiation by the intestinal stem cell niche. Cell Mol Life Sci 68: 25132523.

14. Mizutani K, Yoon K, Dang L, Tokunaga A, Gaiano N (2007) Differential Notch signalling distinguishes neural stem cells from intermediate progenitors. Nature 449: 351-355.

15. Hofmann MC (2008) Gdnf signaling pathways within the mammalian spermatogonial stem cell niche. Mol Cell Endocrinol 288: 95-103.

16. Sharma M, Afrin F, Satija N, Tripathi RP, Gangenahalli GU (2011) Stromalderived factor-1/CXCR4 signaling: indispensable role in homing and engraftment of hematopoietic stem cells in bone marrow. Stem Cells Dev 20: 933-946.

17. Stier S, Ko Y, Forkert R, Lutz C, Neuhaus T, et al. (2005) Osteopontin is a hematopoietic stem cell niche component that negatively regulates stem cell pool size. J Exp Med 201: 1781-1791.

18. Guo X, Wang XF (2009) Signaling cross-talk between TGF-beta/BMP and other pathways. Cell Res 19: 71-88

19. Dejima K, Kanai MI, Akiyama T, Levings DC, Nakato H (2011) Novel contactdependent bone morphogenetic protein (BMP) signaling mediated by heparan sulfate proteoglycans. J Biol Chem 286: 17103-17111.

20. Dellatore SM, Garcia AS, Miller WM (2008) Mimicking stem cell niches to increase stem cell expansion. Curr Opin Biotechnol 19: 534-540.

21. Thomson JA, Itskovitz-Eldor J, Shapiro SS, Waknitz MA, Swiergiel JJ, et al (1998) Embryonic stem cell lines derived from human blastocysts. Science 282: $1145-1147$.
22. Takahashi K, Yamanaka S (2006) Induction of pluripotent stem cells from mouse embryonic and adult fibroblast cultures by defined factors. Cell 126 663-676.

23. Discher DE, Mooney DJ, Zandstra PW (2009) Growth factors, matrices, and forces combine and control stem cells. Science 324: 1673-1677.

24. Dobson CM (2004) Chemical space and biology. Nature 432: 824-828.

25. Barbaric I, Gokhale PJ, Andrews PW (2010) High-content screening of smal compounds on human embryonic stem cells. Biochem Soc Trans 38: 10461050

26. Emre N, Coleman R, Ding S (2007) A chemical approach to stem cell biology Curr Opin Chem Biol 11: 252-258.

27. Chen S, Do JT, Zhang Q, Yao S, Yan F, et al. (2006) Self-renewal of embryonic stem cells by a small molecule. Proc Natl Acad Sci U S A 103: 17266-17271.

28. Ding S, Wu TY, Brinker A, Peters EC, Hur W, et al. (2003) Synthetic smal molecules that control stem cell fate. Proc Natl Acad Sci USA 100: 7632-7637.

29. Wei ZL, Petukhov PA, Bizik F, Teixeira JC, Mercola M, et al. (2004) Isoxazolylserine-based agonists of peroxisome proliferator-activated receptor: design synthesis, and effects on cardiomyocyte differentiation. J Am Chem Soc 126 16714-16715.

30. Wu X, Ding S, Ding Q, Gray NS, Schultz PG (2004) Small molecules that induce cardiomyogenesis in embryonic stem cells. J Am Chem Soc 126: 1590-1591.

31. Watanabe K, Ueno M, Kamiya D, Nishiyama A, Matsumura M, et al. (2007) A ROCK inhibitor permits survival of dissociated human embryonic stem cells. Nat Biotechnol 25: 681-686.

32. Barbaric I, Gokhale PJ, Jones M, Glen A, Baker D, et al. (2010) Nove regulators of stem cell fates identified by a multivariate phenotype screen of small compounds on human embryonic stem cell colonies. Stem Cell Res 5 : 104-119.

33. Desbordes SC, Placantonakis DG, Ciro A, Socci ND, Lee G, et al. (2008) Highthroughput screening assay for the identification of compounds regulating selfrenewal and differentiation in human embryonic stem cells. Cell Stem Cell 2 602-612.

34. Burton P, Adams DR, Abraham A, Allcock RW, Jiang Z, et al. (2010) Erythro9-(2-hydroxy-3-nonyl)adenine (EHNA) blocks differentiation and maintains the expression of pluripotency markers in human embryonic stem cells. Biochem J 432: 575-584.

35. Gonzalez R, Jennings LL, Knuth M, Orth AP, Klock HE, et al. (2010) Screening the mammalian extracellular proteome for regulators of embryonic human stem cell pluripotency. Proc Natl Acad Sci U S A 107: 3552-3557.

36. Zhu S, Wurdak H, Wang J, Lyssiotis CA, Peters EC, et al. (2009) A smal molecule primes embryonic stem cells for differentiation. Cell Stem Cell 4: 416426.

37. Borowiak M, Maehr R, Chen S, Chen AE, Tang W, et al. (2009) Small molecules efficiently direct endodermal differentiation of mouse and human embryonic stem cells. Cell Stem Cell 4: 348-358.

38. Chen S, Borowiak M, Fox JL, Maehr R, Osafune K, et al. (2009) A small molecule that directs differentiation of human ESCs into the pancreatic lineage. Nat Chem Biol 5: 258-265.

39. Willems E, Spiering S, Davidovics H, Lanier M, Xia Z, et al. (2011) Smallmolecule inhibitors of the Wnt pathway potently promote cardiomyocytes from human embryonic stem cell-derived mesoderm. Circ Res 109: 360-364.

40. Danovi D, Falk A, Humphreys P, Vickers R, Tinsley J, et al. (2010) Imaging based chemical screens using normal and glioma-derived neural stem cells. Biochem Soc Trans 38: 1067-1071.

41. Flaim CJ, Chien S, Bhatia SN (2005) An extracellular matrix microarray for probing cellular differentiation. Nat Methods 2: 119-125

42. Flaim CJ, Teng D, Chien S, Bhatia SN (2008) Combinatorial signaling microenvironments for studying stem cell fate. Stem Cells Dev 17: 29-39.

43. Soen Y, Mori A, Palmer TD, Brown PO (2006) Exploring the regulation of human neural precursor cell differentiation using arrays of signaling microenvironments. Mol Syst Biol 2: 37

44. LaBarge MA, Nelson CM, Villadsen R, Fridriksdottir A, Ruth JR, et al. (2009) 
Citation: Brafman DA (2012) Bioengineering of Stem Cell Microenvironments Using High-Throughput Technologies. J Bioengineer \& Biomedical Sci S5:004. doi:10.4172/2155-9538.S5-004

Page 8 of 8

Human mammary progenitor cell fate decisions are products of interactions with combinatorial microenvironments. Integr Biol (Camb) 1: 70-79.

45. Brafman DA, Shah KD, Fellner T, Chien S, Willert K (2009) Defining long-term maintenance conditions of human embryonic stem cells with arrayed cellular microenvironment technology. Stem Cells Dev 18: 1141-1154.

46. Curran JM, Chen R, Hunt JA (2006) The guidance of human mesenchymal stem cell differentiation in vitro by controlled modifications to the cell substrate. Biomaterials 27: 4783-4793.

47. Zhao F, Grayson WL, Ma T, Bunnell B, Lu WW (2006) Effects of hydroxyapatite in 3-D chitosan-gelatin polymer network on human mesenchymal stem cell construct development. Biomaterials 27: 1859-1867.

48. Craiu A, Saito Y, Limon A, Eppich HM, Olson DP, et al. (2005) Flowing cells through pulsed electric fields efficiently purges stem cell preparations of contaminating myeloma cells while preserving stem cell function. Blood 105: 2235-2238.

49. Hayman MW, Smith KH, Cameron NR, Przyborski SA (2005) Growth of human stem cell-derived neurons on solid three-dimensional polymers. J Biochem Biophys Methods 62: 231-240.

50. Anderson DG, Levenberg S, Langer R (2004) Nanoliter-scale synthesis of arrayed biomaterials and application to human embryonic stem cells. Nat Biotechnol 22: 863-866

51. Benoit DS, Schwartz MP, Durney AR, Anseth KS (2008) Small functional groups for controlled differentiation of hydrogel-encapsulated human mesenchymal stem cells. Nat Mater 7: 816-823.

52. Anderson DG, Putnam D, Lavik EB, Mahmood TA, Langer R (2005) Biomateria microarrays: rapid, microscale screening of polymer-cell interaction. Biomaterials 26: 4892-4897.

53. Mei Y, Saha K, Bogatyrev SR, Yang J, Hook AL, et al. (2010) Combinatoria development of biomaterials for clonal growth of human pluripotent stem cells. Nat Mater 9: 768-778.

54. Brafman DA, Chang CW, Fernandez A, Willert K, Varghese S, et al. (2010) Long-term human pluripotent stem cell self-renewal on synthetic polymer surfaces. Biomaterials 31: 9135-9144

55. Hay DC, Pernagallo S, Diaz-Mochon JJ, Medine CN, Greenhough S, et al. (2011) Unbiased screening of polymer libraries to define novel substrates for functional hepatocytes with inducible drug metabolism. Stem Cell Res 6: 92102.

56. Gobaa S, Hoehnel S, Roccio M, Negro A, Kobel S, et al. (2011) Artificial niche microarrays for probing single stem cell fate in high throughput. Nat Methods 8: 949-955.

57. Kobel S, Lutolf M (2010) High-throughput methods to define complex stem cel niches. Biotechniques 48: ix-xxii.

58. Kurth I, Franke K, Pompe T, Bornhäuser M, Werner C (2009) Hematopoietic stem and progenitor cells in adhesive microcavities. Integr Biol (Camb) 1: 427434

59. Lutolf MP, Doyonnas R, Havenstrite K, Koleckar K, Blau HM (2009) Perturbation of single hematopoietic stem cell fates in artificial niches. Integr Biol (Camb) 1 $59-69$

60. Cordey M, Limacher M, Kobel S, Taylor V, Lutolf MP (2008) Enhancing the reliability and throughput of neurosphere culture on hydrogel microwell arrays. Stem Cells 26: 2586-2594

61. Mohr JC, de Pablo JJ, Palecek SP (2006) 3-D microwell culture of human embryonic stem cells. Biomaterials 27: 6032-6042.

62. Karp JM, Yeh J, Eng G, Fukuda J, Blumling J, et al. (2007) Controlling size shape and homogeneity of embryoid bodies using poly(ethylene glycol) microwells. Lab Chip 7: 786-794.

63. Bauwens CL, Peerani R, Niebruegge S, Woodhouse KA, Kumacheva E, et al. (2008) Control of human embryonic stem cell colony and aggregate size heterogeneity influences differentiation trajectories. Stem Cells 26: 2300-2310.

64. Hwang YS, Chung BG, Ortmann D, Hattori N, Moeller HC, et al. (2009) Microwell-mediated control of embryoid body size regulates embryonic stem cell fate via differential expression of WNT5a and WNT11. Proc Natl Acad Sci U S A 106: 16978-16983.

65. van Noort D, Ong SM, Zhang C, Zhang S, Arooz T, et al. (2009) Stem cells in microfluidics. Biotechnol Prog 25: 52-60.

66. Güell M, van Noort V, Yus E, Chen WH, Leigh-Bell J, et al. (2009) Transcriptome complexity in a genome-reduced bacterium. Science 326: 1268-1271.

67. Figallo E, Cannizzaro C, Gerecht S, Burdick JA, Langer R, et al. (2007) Microbioreactor array for controlling cellular microenvironments. Lab Chip 7: 710719

68. Park JY, Kim SK, Woo DH, Lee EJ, Kim JH, et al. (2009) Differentiation of neural progenitor cells in a microfluidic chip-generated cytokine gradient. Stem Cells 27: 2646-2654.

69. Cosson S, Kobel SA, Lutolf ML (2009) Capturing complex protein gradients on biomimetric hydrogels for cell-based assays. Adv Funct Mater 19: 3411-3419.

70. Engler AJ, Sen S, Sweeney HL, Discher DE (2006) Matrix elasticity directs stem cell lineage specification. Cell 126: 677-689.

71. Saha K, Keung AJ, Irwin EF, Li Y, Little L, et al. (2008) Substrate modulus directs neural stem cell behavior. Biophys J 95: 4426-4438.

72. Griffith LG, Swartz MA (2006) Capturing complex 3D tissue physiology in vitro. Nat Rev Mol Cell Biol 7: 211-224.

73. Ou DB, He Y, Chen R, Teng JW, Wang HT, et al. (2011) Three-dimensional co-culture facilitates the differentiation of embryonic stem cells into mature cardiomyocytes. J Cell Biochem 112: 3555-3562.

74. Miki T, Ring A, Gerlach J (2011) Hepatic differentiation of human embryonic stem cells is promoted by three-dimensional dynamic perfusion culture conditions. Tissue Eng Part C Methods 17: 557-568.

75. Yang F, Cho SW, Son SM, Hudson SP, Bogatyrev S, et al. (2010) Combinatoria extracellular matrices for human embryonic stem cell differentiation in 3D. Biomacromolecules 11: 1909-1914.
This article was originally published in a special issue, Emerging Bioengineering Techniques handled by Editor(s). Dr. Partha Roy, "University of Pittsburgh", USA. 